\title{
Correlation of temperature dependence of quasielastic-light-scattering intensity and $\alpha$-relaxation time
}

\author{
S. Kojima \\ Institute of Applied Physics, University of Tsukuba, Ibaraki 305, Japan \\ V. N. Novikov \\ IA\&E, Russian Academy of Sciences, Novosibirsk, 630090, Russia
}

(Received 15 February 1996)

\begin{abstract}
Quasielastic light scattering (QLS) in propylene glycol is measured in a temperature interval 95-320 K in order to study the fast relaxation processes. A model of the fast relaxation is proposed that predicts the growth of the QLS as a function of the free volume. By fitting the experimental data on QLS within the framework of the model, the Vogel-Fulcher-Tammann temperature $T_{0}$ was found. The latter is in good agreement with the value of $T_{0}$ determined from the temperature dependence of the $\alpha$-relaxation time. [S0163-1829(96)02126-1]
\end{abstract}

\section{INTRODUCTION}

A fast $\beta$-relaxation process with a characteristic time of the order of picoseconds has been observed in a number of materials both in Raman and inelastic-neutron-scattering measurements (see, e.g., Refs. 1-8). Recently, indications of this process in dielectric relaxation data were also found. ${ }^{9}$ The main features of the fast relaxation are quite different from those of the main, $\alpha$ relaxation: respective relaxation time depends only slightly on temperature, while the amplitude increases with temperature, especially above the glass transition temperature $T_{g}$; however, at $T>T_{c}$ [where $T_{c}$ is a crossover temperature that for fragile glasses was found to be close to the critical temperature of the mode-coupling-theory $\left.(\mathrm{MCT})^{10}\right]$ the amplitude of the fast relaxation process is temperature independent. ${ }^{7,8}$ Comparisons of the experimental data with the predictions of the MCT show qualitative agreement; however, quantitative deviations were found that were attributed to the contribution of the so-called boson peak, i.e., excess low-frequency vibrations in the spectral region 1 $\mathrm{THz}$, which is close to that of the fast relaxation. ${ }^{11}$ The boson peak vibrations are still not properly accounted for within the frames of the MCT; on the other hand, it is known that for the strong and intermediate glasses the boson peak is an important feature of the low-frequency dynamics. ${ }^{12}$ Moreover, there is evidence of the predominant role of the excess low-frequency vibrations in the origin of the observed fast relaxation process. First, Jäckle, in his review, ${ }^{11}$ already pointed out that for all glasses the depolarization ratio of the Raman spectra is the same for the boson peak and for the quasielastic scattering; this is a nontrivial fact because the depolarization ratio changes for various glasses in the range $0.25-0.75$. Second, as discussed in Ref. 13, inelasticneutron-scattering data shows that the $\mathbf{q}$ dependence of the dynamical structure factor is the same for the boson peak and the quasielastic region. A natural way to take into account these findings is to assume that the observed quasielastic light and neutron spectra are caused by the relaxation of the vibrations, while a direct coupling of the light or neutrons to a relaxational mode is negligible. ${ }^{11}$ Such an approach was realized in a paper of Gotchiyaev et al. ${ }^{3}$ where a coupling of the boson peak vibrations to some unspecified relaxational mode was assumed in order to describe the low-frequency Raman scattering data above the glass transition temperature in glycerol and some other supercooled liquids. Later on this phenomenological model has been applied to both fragile and strong liquids ${ }^{7,8}$ and a reasonable description of the relaxational spectra has been obtained. However, this model is phenomenological; the main parameter of the model, the relaxation strength $\delta(T)$, which is proportional to the coupling constant of the vibrational to the relaxational mode, was determined by the best fit of the experimental results and the nature of the relaxation mode remains unknown.

In the present paper we develop a model in which we specify the relaxational mode that couples to the (quasi)localized vibrations that form the boson peak. This allows us to estimate the parameter $\delta(T)$, which determines the intensity of the quasielastic scattering in the model. ${ }^{3}$ We found that $\delta(T)$ is proportional to the concentration of the free volume; the latter, within the frames of the free-volume model, ${ }^{14,15}$ determines also the temperature dependence of the viscosity $\eta$ and the $\alpha$-relaxation time $\tau \propto \eta / T$. It means that the model predicts some correlation between the $\alpha$ and the fast relaxation in the sense that their temperature dependences, being quite different, are determined by the same parameter. It is known that the Vogel-Fulcher-Tammann temperature $T_{0}$ can be used to describe the temperature dependence of the $\alpha$-relaxation time $\tau_{\alpha}(T) .{ }^{16,17}$ Within the framework of the model, the same temperature $T_{0}$ determines the intensity of the quasielastic light scattering (QLS). We compared our experimental results for the intensity of the quasielastic light scattering in propylene glycol with the literature data on the temperature dependence of the $\alpha$-relaxation time obtained by the dielectric measurements. ${ }^{18,19}$ The value of $T_{0}$ found by fitting the QLS data is in good agreement with that found from the temperature dependence of $\tau_{\alpha}(T)$.

\section{EXPERIMENT}

The sample of propylene glycol of $99.5 \%$ purity was obtained from Wako Chemical Co. Ltd. No further purification was performed. Right-angle Raman spectra have been mea- 


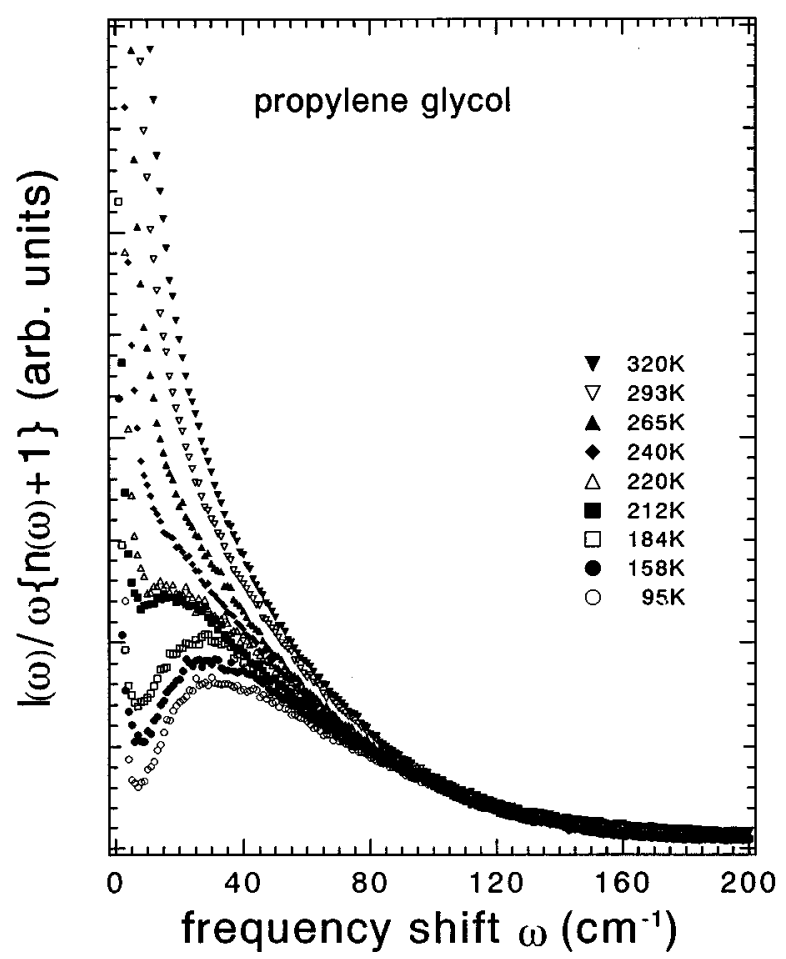

FIG. 1. Reduced Raman spectra of propylene glycol at different temperatures.

sured using a triple monochromator of additive dispersion (Jobin Yvon, T64000) with a spectral resolution of $2 \mathrm{~cm}^{-1}$. The light from an Ar-ion laser with power of $200 \mathrm{~mW}$ was focused into the sample in a quartz optical cell with dimensions of $7 \times 7 \times 12 \mathrm{~mm}^{3}$ through a lens with a focal length of $200 \mathrm{~mm}$. The temperature of the sample was controlled within $\pm 0.5 \mathrm{~K}$. The depolarization ratio was determined by the use of the high-quality prism polarizers with an extinction ratio of $5 \times 10^{-6}$. The scattering area in the sample was always monitored by a television camera with magnification of up to 300 . The monitoring is necessary to confirm that the incindent beam does not cross microbubbles or striae in a glass state. The output signals from the spectrometer were detected by the photon-counting system with a photomultiplier (Hamamatsu Photonics, R464S).

\section{RAMAN SCATTERING SPECTRA}

The temperature dependence of the polarized Raman scattering spectra was determined in both supercooled liquid and a glassy state in the temperature interval $320-95 \mathrm{~K}$ with the glass transition at $172 \mathrm{~K}$. Stokes-side Raman spectra reduced by the temperature factor $\omega[n(\omega)+1]$ with $n(\omega)=[\exp (\hbar \omega / T)-1]^{-1}$ are presented in Fig. 1. All spectra have been normalized by the integrated intensity of the highfrequency mode at $842 \mathrm{~cm}^{-1}$.

The depolarization ratio $\rho(\omega)=I_{V H}(\omega) / I_{V V}(\omega)$ was measured in the same temperature interval. Typical results are shown in Fig. 2. In contrast to the remarkable temperature dependence of the spectra line shapes, it is found that the depolarization ratio is nearly constant in the frequency interval from 5 to $200 \mathrm{~cm}^{-1}$ and is independent of temperature within the experimental error. The value $\rho=0.55$ and the
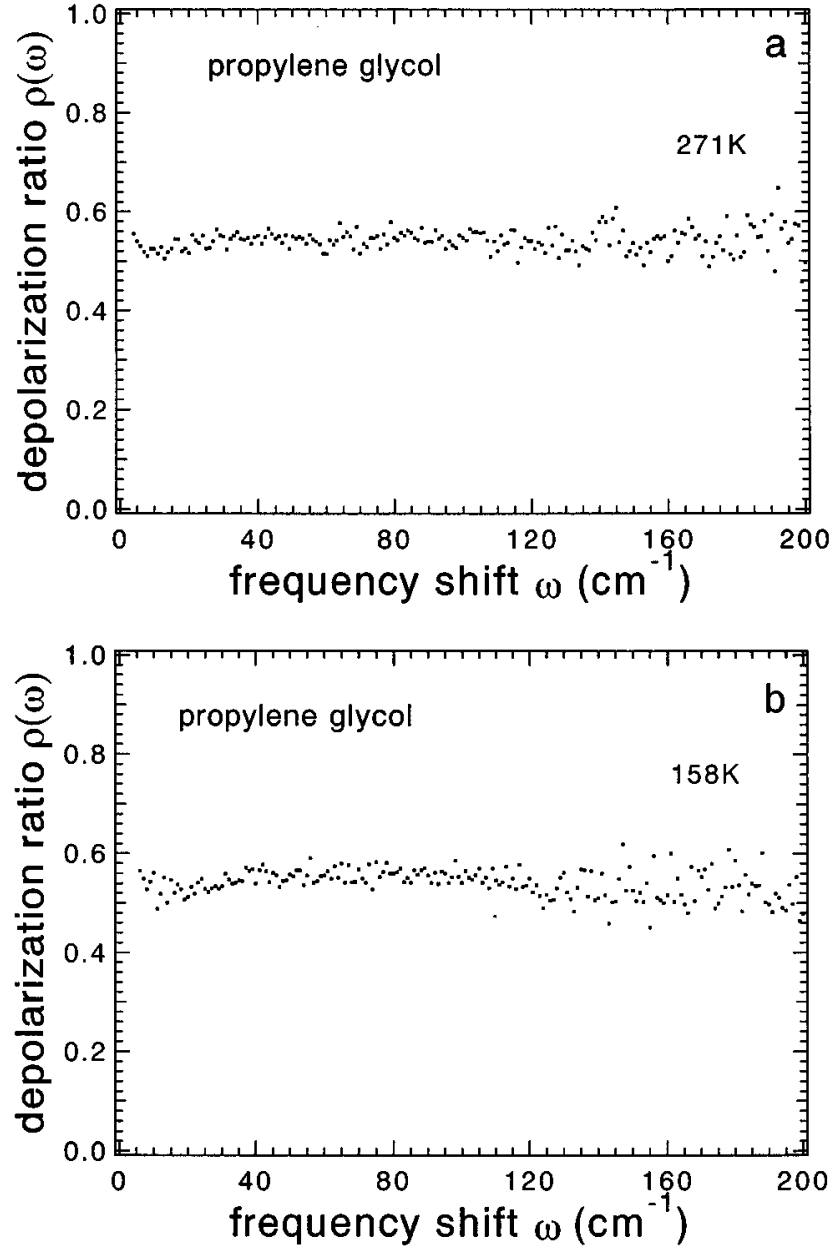

FIG. 2. Frequency dependence of the depolarization ratio in propylene glycol at (a) $271 \mathrm{~K}$ and (b) $158 \mathrm{~K}$.

degree of constancy are almost the same as those of glycerol. $^{3,20}$

\section{MODEL}

As pointed out in the Introduction, there are reasons to believe that the quasielastic light scattering is caused mainly by the vibration relaxation and direct coupling of the light to the relaxation mode is negligible. In this case the reduced scattering intensity $I_{r}(\omega, T)=I(\omega, T) / \omega[n(\omega)+1]$ can be expressed through the susceptibility function of the vibrations at a given temperature and a coefficient that is the respective spectrum when the relaxation is absent, i.e., the lowtemperature spectrum ${ }^{3}$

$$
I_{r}(\omega, T)=\frac{2}{\pi} \int I_{r}(\Omega, 0) \chi^{\prime \prime}(\omega, \Omega) \Omega^{2} d \Omega
$$

where $\chi(\omega, \Omega)$ is the susceptibility of the vibrational eigenmode with a frequency $\Omega$,

$$
\chi(\omega, \Omega)=\frac{1}{\omega^{2}-\Omega^{2}+m(\omega)} .
$$


In Eq. (2) $m(\omega)$ is a relaxation memory function, which, in the case of the simple Debye single-relaxation time approximation, is equal to

$$
m(\omega)=\frac{\delta^{2}}{1+i \omega \tau} .
$$

In Eq. (3) the parameter $\delta(T)$ characterizes the strength of the relaxation process. When $T \rightarrow 0$, also $\delta \rightarrow 0$; as a result, the imaginary part of the susceptibility Eq. (2) is proportional to the $\underline{\underline{\delta}}$ function, $\chi^{\prime \prime}(\omega, \Omega)=\pi \underline{\underline{\delta}}\left(\omega^{2}-\Omega^{2}\right)$, so Eq. (1) becomes an identity. Earlier, Eq. (1) with the memory function Eq. (3) was used to describe the quasielastic scattering in supercooled liquids with $\delta(T)$ and $\tau(T)$ as phenomenological fitting parameters. ${ }^{3,7,8}$

In order to estimate the parameter $\delta(T)$ we need to know the mechanism of the vibration relaxation. We propose a model in which fluctuations of the free volume are responsible for the vibration relaxation. Let us consider a localized harmonic vibration with a frequency $\Omega$ described by a generalized eigenmode $\eta(t)$. The effective harmonic potential $U_{0}(\eta)$ can be written in the form

$$
U_{0}=\frac{1}{2} M \Omega^{2} \eta^{2},
$$

where $M$ is an effective mass of the vibrational mode. Let us consider the influence of the time fluctuations of the free volume on the vibrational mode. Quasilocalized vibrations that form the boson peak have a characteristic localization length of the order of nanometer and typically some tenths or hundreds of atoms participate in these vibrational modes. ${ }^{21-24}$ Therefore, a single act of the local changing of the free volume can lead, typically, only to small relative changes of the effective potential Eq. (4). Let $V$ be the volume occupied by the vibration and $\Delta V_{f}$ be the change of this volume due to the appearance or disappearance of a small fluctuating free volume inside the region occupied by the vibration. A local fluctuation of the volume leads to the shift of the origin of the mode $\eta$. A simple estimation of the shift of the mode $\eta$ is $\Delta \eta=\left(V+\Delta V_{f}\right)^{1 / 3}-V^{1 / 3}$. For $\Delta V_{f} / V \ll 1$ we obtain $\Delta \eta=\Delta V_{f} / 3 V^{2 / 3}$. This expression can be conveniently represented in the form $\Delta \eta=l \Delta V_{f} / 3 V$, where $l=V^{1 / 3}$ is a characteristic length of the vibration. Considering $\Delta \eta$ as an independent fluctuating variable, we see that the effective potential $U_{0}$ is changed as a result of the fluctuation of the free volume: $U_{0}(\eta) \rightarrow U(\eta)=U_{0}(\eta+\Delta \eta)$. Let us introduce a new variable $\xi$, which is the relative fluctuation of the free volume:

$$
\xi=\frac{\Delta V_{f}}{V} .
$$

In terms of $\xi$ and $\eta$ the effective potential $U(\eta, \xi)$ now looks like

$$
U(\eta, \xi)=\frac{1}{2} M \Omega^{2} \eta^{2}+M \Omega^{2}\left(\frac{l}{3}\right) \eta \xi+\frac{1}{2} M \Omega^{2}\left(\frac{l}{3}\right)^{2} \xi^{2} .
$$

The linear in $\eta$ term in Eq. (6) appears due to the fluctuating shift of the origin of the mode $\eta$. The potential Eq. (6) describes a localized harmonic mode $\eta$ interacting with a relaxation mode $\xi$. In order to take into account the thermal fluctuations we have to add in Eq. (6) the term $b \xi^{2} / 2$, which is the minimal work needed to create a given fluctuation of the free volume $\Delta V_{f}$. For Gaussian fluctuations the constant $b$ is proportional to the inverse mean-square value of $\xi^{2}$, $b=T /\left\langle\xi^{2}\right\rangle$, where $T$ is temperature.

The standard methods of the theory of fluctuations ${ }^{25}$ can be used now to obtain the equations of motion for the variables $\eta$ and $\xi$. To this end, let us introduce a dissipation function $F(\xi)$ for the relaxation variable $\xi$ :

$$
F(\dot{\xi})=\frac{1}{2} h \dot{\xi}^{2},
$$

where $h$ is a dissipative constant. Just as in Ref. 3, we neglect the linewidth of the vibrational mode $\gamma_{0}$ due to processes other than the relaxation process under consideration. The equations of motion are determined by the Lagrangian of the system $L, L=K-U$, where the kinetic energy $K$ is determined by the new vibrational mode $\eta, K=M \eta^{2} / 2$, and by the dissipation function $F(\xi)$ :

$$
\frac{d}{d t}\left(\frac{\partial L}{\partial \dot{q}}\right)-\frac{\partial L}{\partial q}=-\frac{\partial F}{\partial \dot{q}},
$$

where $q$ stands for $\eta$ or $\xi$. Using Eqs. (5)-(8) we obtain

$$
\begin{gathered}
M \ddot{\eta}(t)+M \Omega^{2} \eta(t)+M \Omega^{2}\left(\frac{l}{3}\right) \xi(t)=f(t), \\
h \dot{\xi}+\left[M \Omega^{2}\left(\frac{l}{3}\right)^{2}+b\right] \xi(t)+M \Omega^{2}\left(\frac{l}{3}\right) \eta(t)=0 .
\end{gathered}
$$

In Eq. (9) $f(t)$ is the external force caused by the light. As mentioned above, we assume that there is no direct interaction of the light with the relaxational mode $\xi$; the latter influences the light scattering only indirectly via an interaction of the vibrations with the relaxational mode. Taking the Fourier transforms of Eqs. (9) and (10) and denoting $\tau=h /\left[M \Omega^{2}(l / 3)^{2}+b\right]$, we have

$$
\begin{gathered}
\left(-\omega^{2}+\Omega^{2}\right) \eta_{\omega}+\Omega^{2}\left(\frac{l}{3}\right) \xi_{\omega}=\frac{f_{\omega}}{M}, \\
(i \omega \tau+1) \xi_{\omega}+\left(\frac{3 \delta^{2}}{l}\right) \eta_{\omega}=0 .
\end{gathered}
$$

In Eq. (12) we introduced the dimensionless parameter $\delta$, which characterizes the strength of the relaxation; it is defined by the expression

$$
\delta^{2}=\frac{M \Omega^{2} l^{2}}{M \Omega^{2} l^{2}+9 b} .
$$

From Eqs. (11) and (12) it is easy to find the susceptibility of the mode $\eta$ :

$$
\chi(\omega)=\left(\omega^{2}-\Omega^{2}+\frac{\Omega^{2} \delta^{2}}{1+i \omega \tau}\right)^{-1} .
$$

The parameter $\delta(T)$ is of the most importance for the model. Its temperature dependence determines the temperature evolution of the intensity of the quasielastic scattering and softening of the vibrational mode. From Eq. (13) it is obvious that $0<\delta<1$. Let us consider this parameter in more detail. According to Eq. (6), $b=T V^{2} /\left\langle\left(\Delta V_{f}\right)^{2}\right\rangle$. From the fluctua- 
tion theory it is known that $\left\langle(\Delta V)^{2}\right\rangle=æ T V$, where $æ$ is the isothermal compressibility $æ=-V^{-1}(\partial V / \partial P)_{T}$. So it is clear that $\left\langle\left(\Delta V_{f}\right)^{2}\right\rangle$ is an intensive variable $\left\langle\left(\Delta V_{f}\right)^{2}\right\rangle \propto V_{f}$. Let us select the dependence of $\left\langle\left(\Delta V_{f}\right)^{2}\right\rangle$ on $V_{f}$ and $T$ explicitly:

$$
\left\langle\left(\Delta V_{f}\right)^{2}\right\rangle=\mathfrak{x}_{f} T V_{f},
$$

where the coefficient $\mathfrak{x}_{f}$ is defined by this expression and has the sense of the isothermal compressibility of the free volume. Using Eq. (15), one can simplify the expression Eq. (13) for the parameter $\delta$. Since $b=V^{2} / V_{f} \mathfrak{x}_{f}$, we have, for $\delta^{2}$,

$$
\delta^{2}=g /(g+1),
$$

where

$$
g=\rho v^{2} \mathfrak{f}_{f} V_{f} / 9 V .
$$

Here $\rho=M / V$ is the mass density, $v$ is the sound velocity, and we used the relation $\Omega \approx v / l$, which is usually assumed for the low-frequency vibrations in acoustical region. ${ }^{21,22,26}$ From Eqs. (16) and (17) it is clear that $\delta$ and $g$ are frequency-independent parameters whose temperature dependence is determined mainly by that of the free-volume concentration $V_{f}(T) / V$.

Finally, let us write down explicitly the expression for the intensity of the light scattering, which we used to fit the experimental data:

$$
I_{r}(\omega, T)=\frac{2}{\pi} \int \frac{\widetilde{I}_{0}(\Omega) \frac{\delta^{2} \Omega^{2} \gamma}{\omega^{2}+\gamma^{2}} \Omega^{2} d \Omega}{\left\{\omega^{2}-\Omega^{2}+\gamma \frac{\delta^{2} \Omega^{2} \gamma}{\omega^{2}+\gamma^{2}}\right\}^{2}+\omega^{2}\left(\frac{\delta^{2} \Omega^{2} \gamma}{\omega^{2}+\gamma^{2}}\right)^{2}} .
$$

Here $\gamma=\tau^{-1}$ and $\widetilde{I}_{0}(\Omega)$ is the low-temperature spectrum of the boson peak modified in order to take into account the temperature dependence of the elastic constants. This dependence is not accounted for by the relaxation process under consideration. We suppose that with increasing temperature the boson peak can change its position and amplitude due to quasiharmonic softening of the lattice. On the other hand, it is well known that the spectral shape of the boson peak is universal for many materials. ${ }^{21,26}$ So we assume that the boson peak keeps its spectral shape during temperature evolution. The most simple way to formalize this assumption is the following expression for the modified spectrum of the boson peak:

$$
\widetilde{I}_{0}(\Omega)=I_{0}\left[\Omega\left(\frac{\Omega_{0}}{\Omega_{m}}\right)\right]\left(\frac{I_{m}}{I_{0}}\right) .
$$

Here $\Omega_{0}$ and $I_{0}$ are the frequency of the maximum and the amplitude of the boson peak at very low temperature and $\Omega_{m}$ and $I_{m}$ are the respective frequency and amplitude at some temperature $T$.

\section{RESULTS AND DISCUSSION}

In order to find the temperature dependence of the parameter $\delta$ we fitted the experimental curves presented in Fig. 1 using Eq. (18). Four fitting parameters are in the model: the relaxation strength $\delta^{2}$, the position of the boson peak maxi-

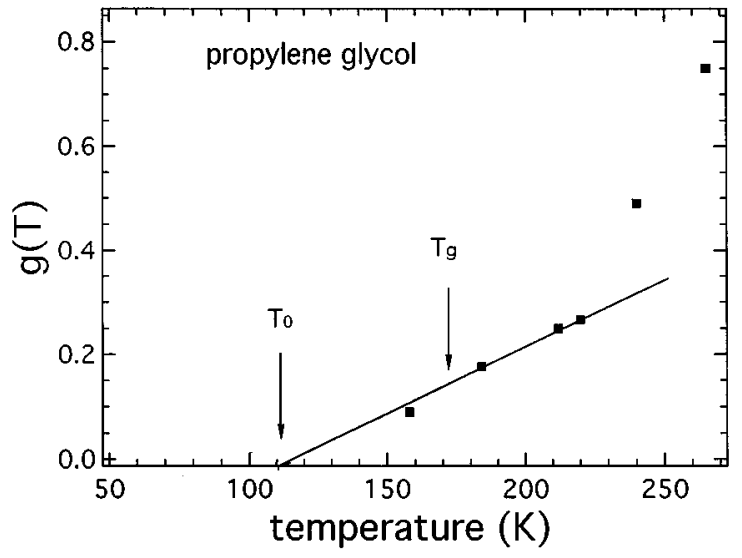

FIG. 3. Temperature dependence of the parameter $g$. The solid line is a fit of the data between $T_{g}$ and $T=220 \mathrm{~K}$.

mum $\Omega_{m}$, the amplitude of the boson peak $I_{m}$, and the width of the quasielastic line $\gamma$. The latter is practically temperature independent in the interval $160-265 \mathrm{~K}$, being equal to $18-20$ $\mathrm{cm}^{-1}$. The results of fitting for the parameters $g=\delta^{2} /\left(1-\delta^{2}\right)$, $\Omega_{m}$, and $I_{m}$ are shown in Figs. 3, 4, and 5, respectively. We preferred to show the parameter $g(T)$ rather than $\delta^{2}(T)$ because $g(T)$ has a more direct connection to the free volume Eq. (17). Let us note that just this parameter $g$ (and not $\delta^{2}$ of the present paper) corresponds to the parameter $\delta^{2}$ of Ref. 27. Before looking more closely at the $g(T)$ behavior, which is the main interest for the present paper, let us make some comments on the temperature dependence of $\Omega_{m}$ and $I_{m}$. As it is clear from Fig. 4, with an increase of temperature the boson peak shifts to lower frequencies nearly linear in temperature; at $\mathrm{T}=158 \mathrm{~K}$, below the glass transition point, the frequency of maximum $\Omega_{0}$ is equal to $39 \mathrm{~cm}^{-1}$, while at $T=265 \mathrm{~K}$ it is more than two times smaller, $\Omega_{m}(T=265$ $\mathrm{K})=17 \mathrm{~cm}^{-1}$. For comparison, in Fig. 4 the temperature dependence of the longitudinal sound velocity is shown (Brillouin scattering data ${ }^{28}$ ). The slope of the $\Omega_{m}(T)$ dependence is 2 times larger than that of the longitudinal sound velocity. This is in qualitative agreement with the results of Ref. 6, where higher softening of the boson peak frequency than that

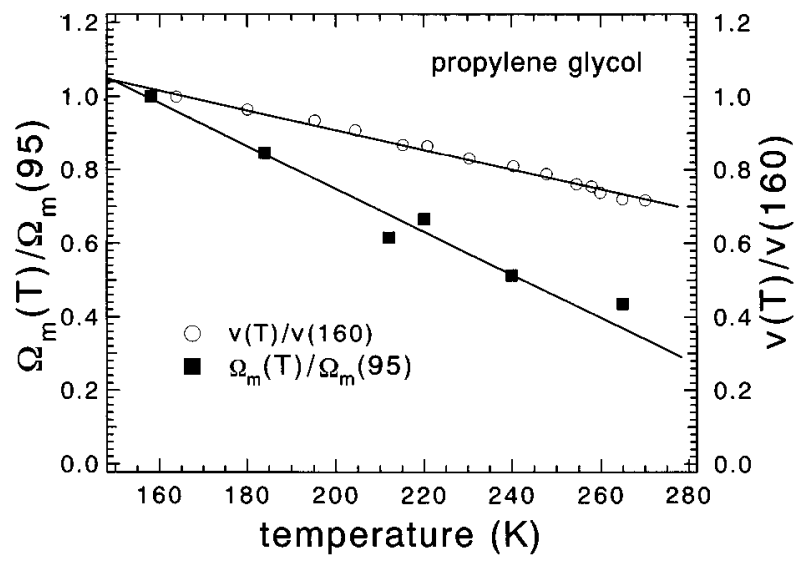

FIG. 4. Temperature dependence of the frequency of the boson peak maximum. For comparison, the Brillouin data for the longitudinal sound velocity is shown (data are from Ref. 28). 


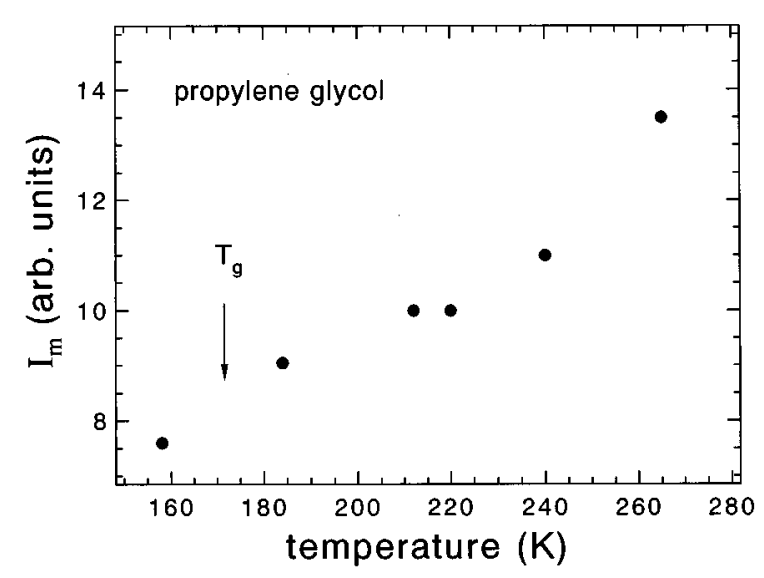

FIG. 5. Temperature dependence of the boson peak amplitude.

of the sound velocity was found in polycarbonate. However, we have no data for the temperature dependence of the transversal sound velocity in propylene glycol, so we cannot make a comparison of $\Omega_{m}$ with the Debye frequency behavior. The intensity of the boson peak increases more or less linearly in temperature below $240 \mathrm{~K}$ (Fig. 5); indications of faster growth are observed at $T>240 \mathrm{~K}$.

Now let us turn to the discussion of the temperature dependence of the relaxation strength parameter $g(T)$ (Fig. 3). This parameter demonstrates practically linear behavior at $T \leqslant 220 \mathrm{~K}$ and a transition to a fast growth in the interval $220-240 \mathrm{~K}$. We interpret this changing of the $g(T)$ dependence as the onset of the $\alpha$-relaxation process. At higher temperatures the $\alpha$ relaxation dominates in the spectral region of the quasielastic scattering. On the other hand, the transition interval 220-240 K strongly correlates with the critical temperature $T_{c}$ defined by MCT. There are different estimations of $T_{c}$ in propylene glycol. Scaling study of dielectric $\alpha$ relaxation by Schönhals et al. ${ }^{18}$ predicts $T_{c}=251.3$ $\mathrm{K}$, while a recent study of the glass transition dynamics by fluorescence measurement of doped chromophores ${ }^{19}$ reported $T_{c}=211.3 \mathrm{~K}$. We also used the fit of high-temperature data for propylene glycol by the MCT formula $\tau_{\alpha}(T) \propto\left(T-T_{c}\right)^{-\gamma}$ to find $T_{c}$. Our fit of the $\tau_{\alpha}(T)$ data of Ref. 19 gives $T_{c}=240 \mathrm{~K}, \gamma=2.5$.

The model of Sec. IV of the present paper predicts that the relaxation parameter $g$ is proportional to the free-volume concentration $V_{f} / V$. The most direct way to check this prediction is to compare the data of Fig. 3 with the positron annihilation data for the free volume. However, as far as we know, there is no such data for propylene glycol. On the other hand, some indirect checking of this result can be obtained by a comparison of the temperature dependence of $g(T)$ with that of the $\alpha$-relaxation time $\tau_{\alpha}(T)$. The reason why such a comparison may be of interest is the following. The free-volume model ${ }^{14,15}$ predicts that the relaxation time of the $\alpha$ process depends on the free volume $V_{f}$ according to the Doolittle equation

$$
\tau_{\alpha}(T)=\tau_{0} \exp \left[A / V_{f}(T)\right]
$$

where $A$ is a constant. So one can compare the temperature dependence of the free volume found from Eq. (20), assum- ing that the $\tau_{\alpha}(T)$ is known, with the $V_{f}(T)$ dependence found from the quasielastic scattering data. We made this comparison using the linearization of the free-volume temperature dependence $V_{f}(T)=a\left(T-T_{0}\right)$, which leads to the Vogel-Fulcher-Tammann equation for the $\alpha$-relaxation time ${ }^{16,17}$

$$
\tau_{\alpha}(T)=\tau_{0} \exp \left[B /\left(T-T_{0}\right)\right],
$$

where $T_{0}$ is the Vogel-Fulcher-Tammann temperature. An important question concerning the fitting of the viscosity or $\tau_{\alpha}(T)$ data by Eq. (21) is what temperature interval one has to use. The recent analysis of Rössler, Hess, and Novikov ${ }^{30}$ and of Stickel et al. ${ }^{31,32}$ shows that there are two characteristic regions in the viscosly behavior of liquids: a lowtemperature region (comparatively close to $T_{g}$ ) and a hightemperature region. For example, these regions are characterized by the different values of the Vogel-FulcherTammann temperature $T_{0}$. In Ref. 30 it is shown that the characteristic temperature that is the boundary between two regimes is close to the critical temperature $T_{c}$ of the modecoupling theory. Taking into account these results, we use the low-temperature value of $T_{0}$ found using data between $T_{g}$ and, roughly, 220-240 K. The fit of the literature data ${ }^{19}$ for $\tau_{\alpha}$ gives $T_{0}=110 \mathrm{~K}$. In Ref. 18 it was found that $T_{0}=117$ $\mathrm{K}$. The linear fit of the QLS data for $g(T)$ in the abovementioned temperature interval gives a value of $T_{0}$ in good agreement with these predictions (Fig. 3). This may be evidence of some correlation of the processes of the slow $\alpha$ relaxation and the fast relaxation in the picosecond domain. One of the possible explanations of such a correlation is given by the present model. Both processes, slow and fast, depend on the free volume: roughly speaking, the slow $\alpha$ process of relaxation is determined by the redistribution of the free volume, while the fast process is connected to some fast relaxational motion that is released by the free-volume holes, so its intensity is proportional to the free-volume content.

\section{CONCLUSION}

The model of quasielastic light scattering proposed in this paper is a further development of the model of Gotchiyaev et $a l .^{3}$ Here, assuming a concrete mechanism of fast relaxation, we can express the phenomenological parameter $\delta^{2}$ of the model $^{3}$ through some other material parameters that can be, in principle, measured independently of quasielastic scattering, namely, the free-volume concentration and isothermal compressibility. Also, the model predicts a connection between temperature dependences of the intensity of the fast relaxation process and the characteristic time of the $\alpha$ relaxation. In spite of the absence of the direct information on the free-volume content, this correlation makes it possible to check the model by a comparison of the Vogel-FulcherTammann temperature found from dielectric relaxation measurements and from the QLS data. The comparison shows good agreement between the two results.

\section{ACKNOWLEDGMENTS}

V.N.N. is grateful to the University of Tsukuba for support during his stay at the Institute of Applied Physics. This work was partially supported by both a Grant in Aid of the Ministry of Education, Science and Culture, No. 06640499,07236208, and INTAS, Grant No. 93-2185. 
${ }^{1}$ G. Winterling, Phys. Rev. B 12, 2432 (1975).

${ }^{2}$ R. Nemanich, Phys. Rev. B 16, 1655 (1977).

${ }^{3}$ V. Z. Gotchiyaev, V. K. Malinovsky, V. N. Novikov, and A. P. Sokolov, Philos. Mag. B 63, 777 (1991).

${ }^{4}$ N. N. Surovtsev, E. Duval, A. Mermet, and V. N. Novikov, J. Phys.: Condens. Matter 8077 (1995).

${ }^{5}$ B. Frick, B. Farago, and D. Richter, Phys. Rev. Lett. 64, 2921 (1990).

${ }^{6}$ U. Buchenau, C. Schönfeld, D. Richter, T. Kanaya, K. Kaji, and R. Wehrmann, Phys. Rev. Lett. 73, 2344 (1995).

${ }^{7}$ E. Rössler, A. P. Sokolov, A. Kisliuk, and D. Quitmann, Phys. Rev. B 49, 14967 (1994).

${ }^{8}$ A. Brodin, D. Engberg, L. M. Torell, L. Börjesson, and A. P. Sokolov, Phys. Rev. B (to be published).

${ }^{9}$ P. Lunkenheimer, A. Pimenov, B. Schiner, R. Böhmer, and A. Lloidl, Europhys. Lett. 33, 611 (1996).

${ }^{10}$ W. Götze and L. Sjögren, Rep. Prog. Phys. 55, 241 (1992).

${ }^{11}$ J. Jäckle, in Amorphous Solids: Low-Temperature Properties, edited by W. A. Phillips (Springer, New York, 1981).

${ }^{12}$ A. P. Sokolov, E. Rössler, A. Kisliuk, and D. Quitmann, Phys. Rev. Lett. 71, 2062 (1994).

${ }^{13}$ B. Frick and D. Richter, Science 267, 1939 (1995).

${ }^{14}$ A. K. Doolittle, J. Appl. Phys. 22, 1471 (1951).

${ }^{15}$ M. Cohen and G. Grest, Phys. Rev. 20, 1077 (1979).

${ }^{16}$ H. Vogel, Phys. Z. 22, 645 (1921).

${ }^{17}$ G. S. Fulcher, J. Am. Ceram. Soc. 8, 339 (1925).
${ }^{18}$ A. Schönhals, F. Kremer, A. Hofmann, and E. W. Fischer, Physica A 201, 263 (1993).

${ }^{19}$ F.-J. Stickel, Ph.D. thesis, Johannes-Gutenberg University, 1995 (unpublished).

${ }^{20}$ S. Kojima, Phys. Rev. B 47, 2924 (1993).

${ }^{21}$ V. K. Malinovsky, V. N. Novikov, P. P. Parshin, A. P. Sokolov, and M. G. Zemlyanov, Europhys. Lett. 11, 43 (1992).

${ }^{22}$ E. Duval, A. Boukenter, and T. Achibat, J. Phys.: Condens. Matter 2, 10227 (1993).

${ }^{23}$ U. Buchenau, Yu. M. Galperin, V. L. Gurevich, and H. R. Shober, Phys. Rev. B 43, 5039 (1993).

${ }^{24}$ H. R. Schober and B. B. Laird, Phys. Rev. B 44, 6746 (1991).

${ }^{25}$ L. D. Landau and E. M. Lifshitz, Statistical Physics (Pergamon, New York, 1980).

${ }^{26}$ V. K. Malinovsky and A. P. Sokolov, Solid State Commun. 57, 757 (1986).

${ }^{27}$ H. Z. Cummins, C. Dreyfus, W. Götze, G. Li, and R. M. Pick, Phys. Rev. E 50, 4847 (1995).

${ }^{28}$ A. Yoshihara, H. Sato, and S. Kojima, Jpn. J. Appl. Phys. (to be published).

${ }^{29}$ J. Y. Yie, T. Hattori, H. Inouye, H. Ueta, H. Nakatsuka, Y. Maruayama, and M. Ishikawa, Phys. Rev. B 53, 8349 (1996).

${ }^{30}$ E. Rössler, K.-U. Hess, and V. N. Novikov (unpublished).

${ }^{31}$ F.-J. Stickel, E. W. Fisher, and R. Richert, J. Chem. Phys. 102, 6251 (1995).

${ }^{32}$ F.-J. Stickel, E. W. Fisher, and R. Richert, J. Chem. Phys. 104, 2043 (1995). 